\title{
Der Mythos des Primats der Preisstabilität. Anmerkungen zur geldpolitischen Strategie der EZB Claus Thomasberger*
}

Die Strategie der Europäischen Zentralbank (EZB) zeichnet sich gegenüber derjenigen anderer Zentralbanken durch ihre eindimensionale Ausrichtung am Ziel eines stabilen Preisniveaus, dem so genannten ,Primat der Preisstabilität‘, aus. Otmar Issing, Chefvolkswirt und Mitglied des Direktoriums der EZB, hat den Vorrang stabiler Preise zu begründen versucht, indem er die Verteilungswirkungen und die Kosten der Inflation, die durch Verzerrungen der relativen Preise, durch eine veränderte reale Kassenhaltung, durch Preisänderungen (Menükosten) sowie durch die Interaktion der Inflation mit den Steuer- und Sozialleistungssystemen verursacht werden, herausstellt (Issing 2000 und 2003). Und er geht noch einen Schritt weiter. Angesichts der breiten Zustimmung, die der Konzeption der EZB zuteil werde, sei es legitim, die Frage umzukehren. »The real question«, so sein Argument, sei heute: "Why not price stability? (Issing 2000: 179, Hervorh. im Orig.)

Ist dies wirklich die Alternative? Die Erfahrungen des 20. Jahrhunderts zeigen, dass sowohl eine Deflation als auch hohe Inflationsraten die Funktionsweise des Marktsystems bedrohen können. Aber weder gibt es einen Konsens darüber, ob die Definition der Preisstabilität in Termini einer Inflationsrate von "unter, aber nahe der Zwei-Prozent-Marke«, wie die offizielle Sprachregelung der EZB nach der Überprüfung der Strategie im Jahr 2003 lautet, angemessen ist, noch kann es als selbstverständlich gelten, dass andere Ziele als nachrangig zu betrachten sind. Damit ist hier nicht nur der Streit zwischen KeynesianerInnen und MonetaristInnen um das Verhältnis von Preisstabilität und Arbeitslosigkeit angesprochen. Für die Geldpolitik im Rahmen der Europäischen Währungsunion (EWU) - eines in der modernen Geschichte beispiellosen Experiments - kommen weitere konkurrierende Zielsetzungen hinzu: Welche Rolle spielen die externen Beziehungen auf der einen, die regionalnationalen Differenzen innerhalb des Währungsraums auf der anderen Seite? Kann sich die EZB-Strategie darauf beschränken, den Euro-Raum schlicht als ein großes und geschlossenes Land zu betrachten und damit nach außen die Wechselkurse wie nach innen die wirtschaftlichen Divergenzen zwischen den Ländern als geldpolitisch irrelevant beiseite zu schieben? Welche Bedeutung kommt der durchschnittlichen Preisentwicklung im EWU-Raum überhaupt zu? Handelt es sich dabei um mehr als eine rechnerische Größe? Und nicht zuletzt: Ist das Primat der Preisniveaustabilität gegenüber konkurrierenden Zielsetzungen wirklich begründet? Oder ist der post-monetaristische Rahmen, innerhalb dessen sich Issings Denken bewegt, der Struktur der EWU schlicht unangemessen?

Das Primat der Preisstabilität hat - so meine Kritik - eine Schieflage und Inflexibilität der europäischen Geldpolitik zur Folge, die es der EZB nicht nur erschwert, ihrer internationalen Verantwortung, sondern auch den unterschiedlichen Bedingungen im Euro-Raum gerecht zu werden. Es wäre vermessen, hier auf wenigen Seiten eine alternative Strategie

\footnotetext{
* Fachhochschule für Technik und Wirtschaft, Berlin.
} 
entwickeln zu wollen. Der Beitrag wird sich darauf beschränken, einige grundlegende Unzulänglichkeiten der strategischen Reflexionen deutlich zu machen.

\section{Euro als internationale Reservewährung und der Wechselkurs}

Der Euro ist, wie die EZB nicht ohne Stolz betont, eine Währung, die als internationale Reservewährung an Bedeutung gewinnt (vgl. Europäische Zentralbank 2003, Geis et al. 2004). Um so erstaunlicher ist es, dass sie glaubt, den Wechselkurs gegenüber US-Dollar und anderen Schlüsselwährungen sowie den internationalen Währungszusammenhang aus ihrer strategischen Konzeption ausklammern zu können. Noch schwerer verständlich wird die strategische Positionierung der EZB, wenn man berücksichtigt, dass sie sich in der Durchführung ihrer Politik de facto "stark am Wechselkurs orientiert" (Heine/Herr 2004: 192).

Tatsache ist, dass die Etablierung des Euro dazu beigetragen hat, dass die Hierarchie der Währungen auf globaler Ebene an Stabilität verloren hat. Zum ersten Mal seit mehr als einem halben Jahrhundert gibt es eine potentielle Alternative zum US-Dollar - man denke an dessen Funktion als internationale Reservewährung, als Medium internationaler Kontrakte oder als internationales Zahlungsmittel. Die Volatilität der Kurse auf dem internationalen Parkett ist Ausdruck dieser sich verändernden Konstellation. Und die kontinuierliche Aufwertung des Euro gegenüber dem US-Dollar seit nahezu vier Jahren hat hier ihre Grundlage.

Im Hinblick auf die Aufwertung des Euro gegenüber dem US-Dollar muss allerdings beachtet werden, dass die US-Zentralbank auf die letzte Rezession mit einer radikalen Senkung der Leitzinsen reagierte, was - neben der hohen Konsumneigung der Haushalte und dem fiskalischen Impuls - zur schnellen Erholung der US-Konjunktur beitrug. Ganz anders ausgerichtet als in den USA war und ist die Wirtschaftspolitik in Europa. Nicht nur der Stabilitätspakt, auch die Rücksichtnahme auf das Inflationsziel von unter zwei Prozent seitens der EZB haben zur Konsequenz gehabt, dass in wichtigen Ländern der EWU bis heute von einem Aufschwung keine Rede sein kann. Die Folge für den Euro-Raum ist wirtschaftliche Stagnation, Arbeitslosigkeit und eine aufwertende Währung, während in den USA die Wirtschaft stark wächst, das Leistungsbilanzdefizit ausufert und der USADollar abwertet.

Soll eine sharte Landung ‘ des US-Dollar verhindert werden, bedarf es einer internationalen Koordination der Geldpolitik - schon aus dem einfachen Grund, weil die USA ihr Leistungsbilanzdefizit nur in dem Umfang begrenzen können, in dem andere (und das gilt auch für einige europäische Länder) ihre Exportüberschüsse reduzieren. Die Wechselkurspolitik, die eine Aufwertung des Euro gegenüber dem US-Dollar ermöglicht und zugleich begrenzt, stellt eine in diesem Zusammenhang notwendige, wenn auch nicht hinreichende Bedingung dar. Erforderlich wäre es darüber hinaus, im Euro-Raum ein stärkeres wirtschaftliches Wachstum und eine entsprechende Importzunahme aus den USA zuzulassen.

Als Zentralbank nicht nur einer regionalen Leitwährung (eine Rolle, die der Deutschen Bundesbank in den I980er und I990er Jahren zukam), sondern der nach dem US- 
Dollar wichtigsten internationalen Ankerwährung kann sich die EZB einerseits ihrer Mitverantwortung für die Stabilität des internationalen Währungssystems nicht wirklich entziehen, andererseits aber, solange sie am >Primat der Preisstabilität ‘ festhält und die Außenflanke nicht systematisch in ihre strategischen Überlegungen einbezieht, auch nicht gerecht werden. Dabei gibt es zumindest zwei theoretische Schulen, an die eine strategische Positionierung anknüpfen könnte. Es sei hier erstens an die Arbeiten des ehemaligen Präsidenten der Deutschen Bundesbank Otmar Emminger erinnert, der die Bedeutung des 'Spannungsfeldes zwischen innerem und äußerem Gleichgewicht ^ für die Politik der Bundesbank von Anfang an betonte (vgl. Emminger 1976), und an weitere in dieser Tradition stehende Arbeiten (vgl. Riese/Spahn I990, Thomasberger 1993); oder, wenn wir noch weiter zurückgehen, an Keynes' »Tract on Monetary Reform « (Keynes I923: I25 ff.), in dem sich dieser, den Niedergang des britischen Empire und der Leitwährungsfunktion des Pfund Sterling vor Augen, explizit mit dem Konflikt zwischen "stability of prices versus stability of exchange" auseinandersetzt. Zweitens wären hier jene Forschungsergebnisse heranzuziehen, die die Möglichkeiten der internationalen Kooperation in den Vordergrund rücken. Gerade wenn man einräumt, dass die Voraussetzungen für eine Rückkehr zu einem System fester, aber anpassungsfähiger Wechselkurse (wie dem Bretton Woods-System) aufgrund der fehlenden Stabilität der Hierarchie der Währungen heute nicht gegeben sind, so kommt den Überlegungen der Etablierung flexiblerer Formen der Kooperation eine zentrale Bedeutung zu. Zu erwähnen wären hier vor allen Dingen Williamsons bzw. Millers `Zielzonen`-Konzeption und darauf aufbauende Arbeiten, die die Perspektive der internationalen Zusammenarbeit nicht auf die Diskussion möglicher Wechselkursarrangements reduzieren, sondern die Frage in den Vordergrund rücken, »how comprehensive macroeconomic policy coordination could be organized" (Williamson/Miller 1987: 64).

\section{Inflationsunterschiede, kumulative Effekte und divergierende Optionen der nationalen Wirtschaftspolitik}

Theoretisch wenig erforscht sind die Konsequenzen, die sich aus den wirtschaftlichen und wirtschaftspolitischen Unterschieden zwischen den verschiedenen Ländern innerhalb eines einheitlichen Währungsraums für die Geldpolitik ergeben. Tatsache ist, dass die Inflationsrate, gemessen in Form des Harmonisierten Verbraucherpreisindex (HVPI), im Durchschnitt der Jahre 1999-2003 in Frankreich I,7 Prozent, in Spanien drei Prozent, in Irland 4,I Prozent, in Deutschland dagegen nur I,2 Prozent betrug. Insgesamt lagen, wie aus Tabelle I ersichtlich wird, die Inflationsraten in Spanien, Holland, Griechenland, Portugal und Irland deutlich über, in Frankreich und Österreich leicht und in Deutschland erheblich unter dem EWU-Durchschnitt. Der deutsche Wert ist, selbst wenn man die Kriterien der EZB zugrunde legt, problematisch, weil eine Inflationsrate von gerade einmal ein Prozent im Jahr 2003 bedeutet, dass - wenn wir das normale Auf und Ab der Inflation im konjunkturellen Zyklus berücksichtigen - in Jahren der Rezession tatsächlich die Gefahr deflationärer Tendenzen heraufbeschworen wird. Anders formuliert: Die Geldpolitik der EZB ist in den letzten Jahren für Deutschland eindeutig zu restriktiv. 
Tabelle I: Inflationsunterschiede (HVPI) im Euro-Raum ${ }^{I}$

\begin{tabular}{l|c|cccccccccccc}
\hline & $\begin{array}{c}\text { EWU } \\
\text { Mittel }\end{array}$ & D & AUT & FR & BEL & FIN & LUX & IT & SP & NL & GR & POR & IRL \\
\hline 1999 & 1,1 & $-0,5$ & $-0,6$ & $-0,5$ & $-0,0$ & 0,2 & $-0,1$ & 0,6 & 1,1 & 0,9 & 1,0 & 1,1 & 1,4 \\
\hline 2000 & 2,1 & $-0,7$ & $-0,1$ & $-0,3$ & 0,6 & 0,9 & 1,7 & 0,5 & 1,4 & 0,2 & 0,8 & 0,7 & 3,2 \\
2001 & 2,3 & $-0,4$ & $-0,0$ & $-0,5$ & 0,1 & 0,4 & 0,1 & 0,0 & 0,5 & 2,8 & 1,4 & 2,1 & 1,7 \\
2002 & 2,3 & $-1,0$ & $-0,6$ & $-0,4$ & $-0,7$ & $-0,3$ & $-0,2$ & 0,3 & 1,3 & 1,6 & 1,6 & 1,4 & 2,4 \\
2003 & 2,1 & $-1,1$ & $-0,8$ & 0,1 & $-0,6$ & $-0,8$ & 0,4 & 0,7 & 1,0 & 0,1 & 1,3 & 1,2 & 1,9 \\
\hline Summe & & $-3,7$ & $-2,1$ & $-1,9$ & $-0,6$ & 0,4 & 1,9 & 2,1 & 5,3 & 5,6 & 6,1 & 6,5 & 10,6 \\
\hline
\end{tabular}

I Abweichungen gegenüber dem EWU-Mittelwert. Alle Angaben in Prozent. In der Tabelle haben über dem Durchschnitt liegende Inflationsraten ein positives, unterdurchschnittliche ein negatives Vorzeichen.

Quelle: Eurostat

Über den Fünfjahreszeitraum summiert ergibt sich, dass sich das Preisniveau in Spanien gegenüber dem Durchschnitt des Euro-Raums um 5,3 Prozent, in Portugal um 6,5 Prozent und in Irland ı,,6 Prozent erhöht hat. In Frankreich ist es gegenüber dem EWU-Durchschnitt um I,9 Prozent und in Deutschland um 3,7 Prozent gefallen. Oder anders ausgedrückt: Gegenüber Deutschland ist das Preisniveau in Frankreich um I, 8 Prozent, in Spanien um neun Prozent und in Irland um I4,3 Prozent gestiegen! Legen solche Zahlen nicht nahe, dass sich die preisliche Konkurrenzfähigkeit der deutschen Anbieter spürbar verbessert, dasjenige insbesondere der spanischen, niederländischen, griechischen, portugiesischen und irischen Anbieter spürbar verschlechtert hat? Wie kommt es dann aber, dass die spanische und die irische Volkswirtschaft - im direkten Gegensatz zur deutschen - überdurchschnittlich wachsen? Das Wachstum des preisbereinigten Bruttoinlandsprodukts (BIP) betrug in dieser Periode in Frankreich 2,2 Prozent, in Spanien 3,2 Prozent und in Irland gar 7,4 Prozent, in Deutschland dagegen nur I, I Prozent.

Die EZB wie die Deutsche Bundesbank tendieren dazu, den Zusammenhang zwischen Inflationsunterschieden und Wachstumsraten als unerheblich beiseite zu schieben (vgl. EZB 1999, Remsperger 2004). Zwei Argumenten kommt dabei eine zentrale Rolle zu. Erstens seien die Inflationsunterschiede in Europa nicht signifikant höher als z.B. in den USA. Und zweitens wird auf den automatischen Ausgleich verwiesen, der durch den Markt bewirkt werde. Binnenmarkt, Währungsunion und verstärkte Preistransparenz förderten eine Angleichung der Inflationsraten. Hinzu käme der stabilisierende Außenhandelseffekt, der zur Folge habe, dass die Länder mit unterdurchschnittlicher Inflation, wie die Verbesserung der Leistungsbilanz Deutschlands zeige, an preislicher Wettbewerbsfähigkeit gewännen.

Wirklich überzeugen kann diese Argumentation nicht. Gegen einen automatischen Ausgleich sprechen die empirischen Befunde, dass die Wachstums- und Inflationsraten auch nach einem halben Jahrzehnt Währungsunion keine Tendenz in Richtung größerer Konvergenz zeigen und dass die Inflationsunterschiede in Europa eine weit höhere regionale Persistenz als z.B. in den USA aufweisen. Hinzu kommt, dass der Vergleich zwischen 
den USA und dem Euro-Raum der Tatsache nicht Rechnung trägt, dass im Euro-Raum verschiedene Ausgleichsmechanismen, die innerhalb eines Landes existieren, nur schwach ausgebildet sind oder gar vollständig fehlen (Lohn-, Einkommens-, Sozial-, Fiskalpolitik, Länderfinanzausgleich). Und schließlich wird die Bedeutung eines kumulativen Effekts unterschätzt, der darin besteht, dass die einheitliche Geldpolitik bei unterschiedlichen Inflationsraten ganz unterschiedliche reale Kosten der Kreditaufnahme für Unternehmen und Haushalte, d.h. unterschiedliche Realzinsen, zur Folge hat. Nehmen wir den mit den Tagen der Geltungsdauer gewichteten Hauptrefinanzierungssatz als Bezugspunkt, so ergibt sich, dass Kreditnehmer in den ersten fünf Jahren der EWU in Frankreich im Mittel einen Realzins von I,6 Prozent, in Spanien von 0,3 Prozent, in Irland von -0,8 Prozent, in Deutschland aber von 2,I Prozent zu zahlen haben. Nicht auf die absolute Höhe, sondern auf die Unterschiede kommt es hierbei an. Entscheidend ist, dass der formal einheitliche Zins nicht gleiche Bedingungen hervorbringt, sondern umgekehrt die Divergenzen verstärkt: In Ländern mit überdurchschnittlichen Inflationsraten herrschen geldpolitisch expansivere Bedingungen als in Ländern mit größerer Preisstabilität.

Tabelle 2: Realzinsen im Euro-Raum

\begin{tabular}{l|cccccccccccc}
\hline Jahr & D & AUT & FR & BEL & FIN & LUX & IT & SP & NL & GR & POR & IRL \\
\hline 1999 & 2,1 & 2,2 & 2,1 & 1,6 & 1,4 & 1,7 & 1,0 & 0,5 & 0,7 & 0,6 & 0,5 & 0,2 \\
\hline 2000 & 2,6 & 2,0 & 2,2 & 1,3 & 1,0 & 0,2 & 1,4 & 0,5 & 1,7 & 1,1 & 1,2 & $-1,3$ \\
2001 & 2,4 & 2,0 & 2,5 & 1,9 & 1,6 & 1,9 & 2,0 & 1,5 & $-0,8$ & 0,6 & $-0,1$ & 0,3 \\
2002 & 1,9 & 1,5 & 1,3 & 1,6 & 1,2 & 1,1 & 0,6 & $-0,4$ & $-0,7$ & $-0,7$ & $-0,5$ & $-1,5$ \\
2003 & 1,3 & 1,0 & 0,1 & 0,8 & 1,0 & $-0,2$ & $-0,5$ & $-0,8$ & 0,1 & $-1,1$ & $-1,0$ & $-1,7$ \\
\hline Mittelwert & 2,1 & 1,7 & 1,6 & 1,4 & 1,2 & 0,9 & 0,9 & 0,3 & 0,2 & 0,1 & 0,0 & $-0,8$ \\
\hline
\end{tabular}

Quellen: Eurostat und EZB

Hinzu kommt, dass die EZB-Argumentation die unterschiedlichen wirtschaftspolitischen Orientierungen innerhalb der Länder des Euro-Raums unberücksichtigt lässt. Es muss hier genügen, zwei Extreme, die Orientierung am Ziel der Verbesserung der internationalen Wettbewerbsfähigkeit und die binnenorientierte Wachstumsorientierung, einander gegenüberzustellen. Um mit ersterer zu beginnen: Seit dem Zweiten Weltkrieg gelten eine stabile Währung, Exportüberschüsse und eine positive Leistungsbilanz in Deutschland als Garant des wirtschaftlichen Wachstums. Der Geldpolitik der Deutschen Bundesbank fiel in diesem Politikmodell die Aufgabe zu, die deutsche Position außenwirtschaftlich abzusichern, d.h. eine reale Aufwertung der DM auf oder über ihr Gleichgewichtsniveau zu verhindern.

Nun gibt es gute Gründe dafür, dass eine solche Orientierung mit dem Übergang zur EWU ihre Voraussetzungen verloren hat. Aber auch wenn diese Einschätzung richtig ist, so kann doch nicht ausgeschlossen werden, dass einflussreiche Kreise aus Wirtschaft, Politik und Wissenschaft - und sei es nur aus einem falsch verstandenen mikroökonomischen Kalkül heraus - an dieser Logik festhalten. Die überragende Bedeutung, die Forderungen 
nach Senkung der (Lohn-)Kosten, nach Arbeitszeitverlängerung, nach einem Abbau der sozialen Leistungen etc. zur Verbesserung der 'Standortbedingungen ' in der wirtschaftspolitischen Diskussion seit Ende der I990er Jahre hierzulande zukommt (vgl. Thomasberger 1998), deute darauf hin, dass die Verbesserung der internationalen Wettbewerbsposition in der deutschen Wirtschaftspolitik nach wie vor die dominante Orientierung ist. Denn eine überlegene preisliche Wettbewerbsfähigkeit lässt sich, wenn keine nominalen Wechselkurse mehr existieren, nur dadurch sichern, dass der Anstieg der nominalen Kostenund Inflationsraten unter dem Durchschnitt in der Euro-Zone gehalten wird.

Allerdings übersieht das Festhalten an der geschilderten Ausrichtung einen wesentlichen Unterschied zu früher: Im Falle nationaler Währungen bedingt die Existenz von Währungsmärkten, dass die Zinsraten in den verschiedenen Ländern aufgrund ungleicher Liquiditätsprämien, Auf- bzw. Abwertungserwartungen etc. im Finanzmarktgleichgewicht differieren. Daraus resultierte, dass die DM-Zinsraten (als der Leit- bzw. Ankerwährung des EWS) in den späten I980er und den I990er Jahre nicht nur nominal, sondern auch real unter denen der Partnerwährungen lagen, d.h. dass die Bundesbank eine Geldpolitik verfolgte, die expansiver als die der übrigen EWS-Länder war (vgl. Thomasberger 1993). Unter den Bedingungen einer einheitlichen Währung ist dies, wie oben gesehen, eben genau umgekehrt.

Aus dieser Perspektive betrachtet, setzt die Option der Verbesserung der internationalen Wettbewerbsfähigkeit darauf, dass sich in einer Währungsunion letztendlich der aus dem Gewinn an preislicher Wettbewerbsfähigkeit resultierende positive Effekt gegenüber den negativen Folgen der restriktiveren monetären Bedingungen durchsetzt. Zweifel an den Erfolgsaussichten sind angebracht: Betrachten wir die bundesdeutsche Wirtschaft, so werden trotz der bedeutenden Rolle des Exports nur etwa 36 Prozent des BIP exportiert. Der Anteil ist zwar ansteigend, die Zunahme wird aber auf der anderen Seite von der stagnierenden Binnennachfrage - wobei der private Konsum alleine knapp 6o Prozent der Gesamtnachfrage ausmacht - mehr als ausgeglichen. Zusätzlich ist zu berücksichtigen, dass der größere Teil der Exporte in Länder außerhalb der EWU geht. Daher besteht immer die Gefahr, dass der Vorteil, der gegenüber den EWU-Partnern erreicht wird, durch eine Aufwertung des Euro im internationalen Kontext überkompensiert wird.

Tabelle 3: Der deutsche Export I999 bis 2003

\begin{tabular}{|c|c|c|c|}
\hline & \multirow[t]{2}{*}{$\begin{array}{l}\text { Export am } \\
\text { BIP (VGR) }\end{array}$} & \multicolumn{2}{|c|}{$\begin{array}{c}\text { Außenhandel } \\
\text { nach Ländergruppen }\end{array}$} \\
\hline & & EWU & Nicht-EWU \\
\hline 1999 & $29,7 \%$ & $45,1 \%$ & $54,9 \%$ \\
\hline 2000 & $33,8 \%$ & $44,3 \%$ & $55,7 \%$ \\
\hline 2001 & $35,2 \%$ & $43,2 \%$ & $56,8 \%$ \\
\hline 2002 & $36,1 \%$ & $42,4 \%$ & $57,6 \%$ \\
\hline 2003 & $36,1 \%$ & $43,4 \%$ & $56,6 \%$ \\
\hline
\end{tabular}

Quelle: Deutsche Bundesbank 
Es ist daher auch in den nächsten Jahren kaum zu erwarten, dass die deutsche Wachstumsschwäche durch einen steigenden Export nach Spanien oder Irland - oder andere EWUPartner - überwunden werden könnte, selbst wenn die preisliche Wettbewerbsfähigkeit gegenüber diesen weiter gesteigert werden sollte. Nach Spanien gehen gerade fünf Prozent, nach Irland noch nicht einmal o,6 Prozent der bundesdeutschen Exporte! Und alle EWULänder zusammen nehmen weniger als I6 Prozent des bundesdeutschen BIP auf.

Den Gegenpol zur Verbesserung der internationalen Wettbewerbsfähigkeit stellt eine wirtschaftspolitische Orientierung dar, die in erster Linie auf das binnenorientierte Wachstum setzt. Spanien, Portugal und Irland mögen als Beispiele dienen. Die dynamischen Aspekted.h. die Wirkungen divergierender Raten der Preisveränderung, im Gegensatz zu den statischen Effekten, die Preisniveauunterschiede mit sich bringen - stehen hier im Vordergrund. Die niedrigen Realzinsen, die aus den über dem Durchschnitt in der Euro-Zone liegenden Inflationsraten resultieren, und die daraus resultierende expansive Ausrichtung der Geldpolitik werden als Hebel eingesetzt, um Investitionen, Wachstum und Beschäftigung innerhalb des Landes zu fördern. Betrachtet man die vergangenen Jahre, so hat sich die binnenorientierte Wachstumsorientierung als die eindeutig erfolgreichere Konzeption erwiesen. Sie ist auch für den Euro-Raum insgesamt weniger problematisch als die Zielsetzung der Verbesserung der preislichen Wettbewerbsfähigkeit, weil sie nicht darauf setzt, die Vorteile, die die heimische Wirtschaft erzielen mag, auf Kosten der Partnerländer zu erreichen (d.h. keinen merkantilistischen Grundton aufweist), sondern die ökonomische Entwicklung des Euro-Raums in seiner Gesamtheit stützt.

Wirtschaftspolitik im umfassenden Sinne wird in Europa immer noch als eine nationale Angelegenheit betrachtet. Wie steht die EZB zu dieser Teilung der Zuständigkeiten? Wäre es nicht nur aus der Perspektive einer stärkeren Wachstumsorientierung, sondern auch aus jener der Schaffung eines einheitlichen Währungsraums notwendig, das unkoordinierte Nebeneinander von europäischer Geld- und nationaler Wirtschaftspolitik zu überwinden? Und ist es angesichts der ökonomischen und wirtschaftspolitischen Divergenzen angemessen, wenn sich die EZB in ihrer Zielsetzung an einem arithmetischen Durchschnitt orientiert, ohne die unterschiedlichen Orientierungen, Schwerpunktsetzungen und wirtschaftspolitischen Kalküle in ihre Betrachtung einzubeziehen? Hält sie einen einheitlichen Zinssatz für ausreichend, um die einheitliche wirtschaftliche Entwicklung innerhalb des Währungsraums zu gewährleisten? Sollte sie sich nicht - bevor es zu spät ist und bevor die wirtschaftliche Entwicklung noch weiter auseinander läuft - kritisch mit den Orientierungen einzelner Länder auseinandersetzen? Mit der Erweiterung der Währungsunion nach Zentralosteuropa werden die Divergenzen weiter wachsen. Die realwirtschaftlichen Unterschiede - man denke nur an die Produktivität und das Einkommensniveau - erreichen mit der im Mai 2004 vollzogenen Erweiterung der Europäischen Union eine Stufe, auf die die EWU nicht vorbereitet ist. Die strategischen Überlegungen der EZB konzentrieren sich bislang darauf, von den Beitrittsländern die Einhaltung jener Kriterien einzufordern, die die Gründungsmitglieder der EWU in der zweiten Hälfte der I99oer Jahre zu erfüllen hatten. Die unterschiedlichen wirtschaftlichen Ausgangsbedingungen in den zentralosteuropäischen Ländern bleiben dabei unberücksichtigt. Auf die Frage, wie der potentielle Konflikt zwischen nomi- 
naler und realer Konvergenz aufgefangen werden kann, bleibt die EZB bisher eine überzeugende Antwort schuldig.

\section{Das Primat der Preisstabilität - ein Mythos?}

Einerseits stecken die Ungleichgewichte der Weltwirtschaft, die kumulativen Effekte und die unterschiedlichen wirtschaftspolitischen Orientierungen zwischen Ländern des EuroRaums den Rahmen ab, innerhalb dessen sich die EZB bewegt. Andererseits trägt die theoretische Konzeption, auf die sich die europäische Zentralbank stützt, den Bedingungen einer Währungsunion verschiedener Länder in einer komplexen Welt in keiner Weise Rechnung. Mit einer Zielformulierung in Termini einer Inflationsrate unter, aber nahe der Zwei-ProzentMarke ist nichts gewonnen. Die Autonomie der EZB mag die Möglichkeit einschließen, sich der direkten politischen Einflussnahmen seitens der nationalen Regierungen zu entziehen. Aber Autonomie bedeutet nicht, sich gegenüber den wirtschaftlichen Verflechtungen dieser Welt abschotten zu können.

Nun kann man einwenden, dass die geldpolitische Strategie einer Zentralbank in erster Linie der Außendarstellung diene, d.h. die EZB in der Durchführung ihrer Politik die Komplexität der faktischen Bedingungen weitgehend berücksichtige. Sicher ist dieses Argument nicht ganz falsch. Aber es bleibt das Unbehagen, dass durch eine solche Trennung nicht nur das Niveau der theoretisch-konzeptionellen Diskussion negativ beeinflusst wird, sondern auch die Durchführung darunter leidet, wenn strategische Dimensionen nur ad hoc, d.h. in planloser und unsystematischer Weise, in die Geldpolitik Eingang finden. Auch wenn es ein Mythos ist, so beherrscht das Primat der Preisstabilität nicht nur das theoretische Denken der EZB, sondern beeinflusst auch ihre geldpolitische Praxis.

\section{Literatur}

Emminger, Otmar (1976): Deutsche Geld- und Währungspolitik im Spannungsfeld zwischen innerem und äußerem Gleichgewicht, in: Deutsche Bundesbank (Hg.), Währung und Wirtschaft in Deutschland I876-1975, Frankfurt a.M., S. 485-554

Europäische Zentralbank (2003): The International Role of the Euro: Main Developments Since the Inception of Stage Three of Economic and Monetary Union, in: Monthly Bulletin, November, S. 7I-9

Geis, André/Mehl, Arnaud/Wredenborg, Stefan (2004): The International Role of the Euro, Occasional Paper Series, No. I8, Frankfurt a.M.: EZB

Heine, Michael/Herr, Hansjörg (2004): Die Europäische Zentralbank, Marburg Issing, Otmar (2000): Why Price Stability?, in: García Herrero, Alicia/Gaspar, Vítor/Hoogduin, Lex/Morgan, Julian/Winkler, Bernhard (Hg.): Why Price Stability? (First ECB Central Banking Conference) November, Frankfurt a.M.: EZB, S. I79-202

Issing, Otmar (2003) (Hg.): Background Studies for the ECB's Evaluation of its Monetary Policy Strategy, Frankfurt a.M.: EZB

Keynes, John M. (1923): A Tract on Monetary Reform, The Collected Writings of J. Maynard Keynes, Bd. IV, London/Basingstoke: Macmillan 
Remsperger, Hermann (2004): Inflationsdifferentiale im Euro-Raum: Ursachen und Konsequenzen, Heidelberg

Riese, Hajo/Spahn, Heinz-Peter (1990) (Hg.): Geldpolitik und ökonomische Entwicklung, Studien zur monetären Ökonomie 4, Regensburg

Thomasberger, Claus (1993): Europäische Währungsintegration und globale Währungskonkurrenz, Tübingen

Thomasberger, Claus (1998): , Internationale Wettbewerbsfähigkeit: Sinn und Unsinn einer wirtschaftspolitischen Konzeption, Bonn

Williamson, John/Miller, Marcus 1987: Targets and Indicators: A Blueprint for the International Coordination of Economic Policy, Policy Analysis in International Economics, No. 22, Washington D.C.: Institute for International Economics

\section{Ein Schritt vor und zwei Schritte zurück? Zur Kritik an den Vorschlägen der Europäischen Kommission zur Strukturförderung nach 2006 Katharina Erdmenger ${ }^{*}$, Astrid Ziegler ${ }^{\circ}$}

\section{Einleitung}

Im Juli 2004 veröffentlichte die Europäische Kommission ihre Vorschläge für die Verordnungen über den Europäischen Fonds für regionale Entwicklung, den Europäischen Sozialfonds und den Kohäsionsfonds (vgl. Kommission der Europäischen Gemeinschaften 2004), die Grundlage für die Gestaltung der Strukturfonds in der neuen Förderperiode ab 2007 sein werden. Damit ist das europäische Entscheidungsverfahren über die künftige Gestaltung der Strukturförderung eröffnet, es soll im Laufe des Jahres 2005 mit einer Einigung der 25 Mitgliedstaaten und der europäischen Institutionen abgeschlossen werden. Es wäre nun zu vermuten, dass im Mittelpunkt der aktuellen Debatte über die Zukunft der Strukturförderung die von der Kommission vorgeschlagenen Förderinhalte in einer erweiterten EU der 25 stehen würden. Tatsächlich wird diese Diskussion aber um die Frage geführt, wie viel Geld in den europäischen Haushalt für die Strukturförderung eingezahlt werden muss und wie viel die einzelnen Mitgliedstaaten davon erhalten sollen.

EU-Nettozahler - allen voran Deutschland - bestehen darauf, das Volumen ihrer Einzahlungen in den EU-Haushalt insgesamt auf ein Prozent ihrer Wirtschaftsleistung zu begrenzen. ${ }^{\mathrm{I}}$ Die anderen Mitgliedsländer wollen an der bisherigen Regelung (I,I4 Prozent des

* DGB-Bundesvorstandsverwaltung, Berlin.

- Wirtschafts- und Sozialwissenschaftliches Institut (WSI) in der Hans Böckler Stiftung, Düsseldorf.

I EU-Nettozahler, die sich der deutschen Forderung angeschlossen haben, sind Österreich, Frankreich, Großbritannien, die Niederlande und Schweden. 